\title{
Scire mori. Tre exempla virtutis fra Seneca \\ e Silio Italico
}

Diletta Vignola

(University of Genoa)

\section{Scire mori. Three exempla virtutis in Seneca and Silius Italicus}

\begin{abstract}
The purpose of this paper is to offer a new perspective on Silius Italicus' alleged stoicism through an analysis of three episodes in his poem which deal with exemplary deaths: the execution of the so-called "smiling slave" (a Hispanic slave who bears his torments heroically, mocking his torturers, Sil.1.169-181), the death of Marcus Atilius Regulus at Carthage (Sil. $2.340-344 ; 2.435-436 ; 6.535-544$ ), and the death of Lucius Aemilius Paullus at Cannae (Sil. 10.260-288).

My argument is that in Silius' poem both Regulus' and the slave's deaths are modelled on similar passages in Seneca's oeuvre, while the description of Paullus' death, despite being modelled on Livy's account (Liv. 22.49.6-11), includes several philosophical (and especially Senecan) motifs, so that the consul of Cannae becomes a sort of stoic sapiens.
\end{abstract}

\section{Keywords}

Silius Italicus; Punica; Seneca; exemplum virtutis; stoicism; Regulus; Lucius Aemilius Paullus

\section{Acknowledgement}

Vorrei ringraziare Gabriella Moretti per gli spunti di riflessione che mi ha offerto durante la stesura di questo lavoro, nonché per averne letto una bozza preliminare: resta inteso che ogni errore o mancanza è da attribuirsi unicamente a me. La mia gratitudine va inoltre agli anonimi referee, che con i loro suggerimenti hanno contribuito a migliorare notevolmente il lavoro. 


\section{Introduzione}

Che Silio Italico sia considerato un autore stoico - anzi, il più stoico fra i tre epici flavi - non è una novità: già nel 1966, ad esempio, Edward Bassett aveva proposto di vedere in Scipione - ritenuto il vero eroe dei Punica - il diretto erede di un Ercole definito «the 'patron saint' of the Stoics».1

Negli anni ottanta, Margarethe Billerbeck ha tuttavia ridimensionato l'interpretazione rigorosamente stoica del poema siliano proposta da Bassett, facendo notare come molti elementi che questi attribuiva all'afflato filosofico siano da ricondursi più semplicemente alla tradizione romana, e nello stesso tempo ha ridefinito con maggiore accuratezza l'orizzonte filosofico in cui si muove il poeta dei Punica, proponendo in ultima analisi di accostare Silio alla rilettura moderata dello stoicismo operata da Panezio e da Cicerone - tendenza che l'autrice ritiene predominante in età flavia -, piuttosto che allo stoicismo "cinicizzante" predominante invece sotto Claudio e Nerone. ${ }^{2}$

Partendo dalle conclusioni della Billerbeck, che ritengo sul piano generale tuttora le più valide ed equilibrate, vorrei però ora provare a indagare se in qualche specifico passo dei Punica possa essere riconosciuta una ripresa del modello stoico propriamente senechiano e, in tal caso, in che modo le ragioni della filosofia si intersechino con quelle della letteratura. In tale ottica, proverò a rileggere tre episodi del poema - la morte fra i tormenti dello schiavo di Tago, la fine di Marco Atilio Regolo a Cartagine e quella di Lucio Emilio Paolo a Canne - mettendoli a confronto con episodi analoghi o simili tratti dalle opere morali senechiane.

Sebbene infatti in generale non sia facile - anzi, sarebbe quasi impossibile - stabilire con certezza in che misura la matrice di questi exempla $a^{3}$ possa effettivamente essere ricondotta allo stoicismo del poeta, e non piuttosto al semplice recupero della tradizione romana, pure mi sembra che nel caso specifico di questi tre episodi, a differenza di altri potenzialmente simili, tanto la particolare enfasi su temi cari alla dottrina stoica - primi fra tutti la patientia del saggio e la sua capacità di ben morire -, quanto il recupero di-

1 Bassett (1966: p. 259).

2 Billerbeck (1986b). Sullo stoicismo di Silio v. anche von Albrecht (in particolare von Albrecht 1964: pp. 55-86 e passim per la presenza di elementi stoici nei Punica; von Albrecht 1999: pp. 291-292 per la personale adesione - o vicinanza - del poeta allo stoicismo); Billerbeck (1986a: pp. 3134-3143), Matier (1990). Inoltre, in generale sul rapporto tra esemplarità e mos maiorum v. Hölkeskamp (1996) e Stemmler (2000), e più in dettaglio sull'esemplarità nei Punica v. Tipping (2010: pp. 7-13).

3 Tale approccio richiede una definizione preliminare di ciò che si considererà come exemplum. L'exemplum è definito da Gazich (2003), in uno studio dedicato all'epistolario pliniano, come «un meccanismo logico-retorico [...] che è in grado di trasformare in facta e dicta significativi per la comunità semplici eventi personali o persino fatti banali della quotidianità». Questa definizione, pur non potendo essere mutuata in toto per i Punica - giacché in questo caso gli exempla sono tratti non da eventi strettamente personali, ma da eventi bellici, che coinvolgono spesso personaggi di spicco e che hanno dunque un'importanza intrinseca per la comunità tutta - può comunque essere adattata al caso del poema siliano: si considererà qui come exemplum una vicenda che, a prescindere dalla sua rilevanza storica, viene rielaborata e trasformata dal poeta in modo da renderla significativa in quanto testimonianza - implicita o esplicita - di un particolare valore morale. Cfr. anche Hölkeskamp (1996: pp. 312-315) e Stemmler (2000: pp. 168-179). 
retto di motivi e lessico propriamente senechiani possano garantire la legittimità di una lettura (anche) filosofica.

1. Il primo di questi exempla si trova appena qualche centinaio di versi dopo l'inizio del poema: dopo la morte del vecchio Amilcare, il comando delle operazioni viene affidato provvisoriamente al genero Asdrubale, il quale, distinguendosi per la sua particolare crudeltà (vv. 144-148), fa uccidere lo spagnolo Tago per poi farne appendere il corpo martoriato (vv. 148-154). Uno dei suoi schiavi, allora, vedendone la salma oltraggiata, fa irruzione nel palazzo e uccide Asdrubale (vv. 165-168). I Cartaginesi, anch'essi contraddistinti proprio come il loro capo da una spiccata crudeltà, decidono di vendicarlo, e sottopongono il servo fedele ai più raffinati tormenti. Nonostante ciò, egli rimane imperturbabile: ${ }^{4}$

at Poeni, succensa ira turbataque luctu

et saeuis gens laeta, ruunt tormentaque portant.

non ignes candensque chalybs, non uerbera passim

ictibus innumeris lacerum scindentia corpus,

carnificaeue manus penitusue infusa medullis

pestis et in medio lucentes uulnere flammae

cessauere. ferum uisu dictuque, per artem

saeuitiae extenti, quantum tormenta iubebant,

creuerunt artus, atque omni sanguine rupto

ossa liquefactis fumarunt feruida membris.

mens intacta manet. superat ridetque dolores

spectanti similis, fessosque labore ministros

increpitat dominique crucem clamore reposcit.

(Sil.1.169-181)

Certamente la vicenda in questione doveva avere una base storica, e ciò è testimoniato da Livio:

Barbarus eum quidam palam ob iram interfecti ab eo domini obtruncat; comprensusque ab circumstantibus haud alio quam si euasisset uoltu, tormentis quoque cum laceraretur, eo fuit habitu oris ut superante laetitia dolores ridentis etiam speciem praebuerit.

(Liv. 21.2.6)

L'episodio siliano, dunque, ad una prima lettura potrebbe essere interpretato come una versione poetica e, diremmo oggi, "romanzata" della vicenda storica liviana. ${ }^{5}$ Eppure, un

4 Il testo dei Punica, qui e in seguito, è citato dall'edizione di Delz (1987). Per le opere di Seneca, si fa riferimento invece all'edizione delle Epistole e dei Dialoghi di Reynolds (rispettivamente 1965 e 1977) e a quella delle Tragedie di Zwierlein (1986).

5 Del resto, come osserva Langlands (2018: pp. 218-219), in Livio - che costituisce per noi la fonte più antica sull'episodio - la vicenda dello schiavo non sembra avere uno specifico valore esemplare. 
dettaglio colpisce in modo particolare: mentre Livio parla genericamente di tormenta, Silio sembra insistere in modo quasi parossistico sulla crudeltà e sull'ingegnosità della pena, con una dovizia di particolari che non si trova neanche nelle altre due fonti che abbiamo sull'episodio, ossia Valerio Massimo (Val. Max. 3.3.ext.7) e Giustino (Iustin. 44.2.4).

Come è stato osservato già da Danesi Marioni e poi da Vinchesi, ${ }^{6}$ tuttavia, per questo aneddoto potremmo disporre anche di un'ulteriore fonte, che invece sul tipo di supplizio risulta molto più accurata. Seneca, infatti, pur non facendo mai il nome né di Tago né di Asdrubale, ci offre un resoconto molto vicino a quello riproposto da Silio: ${ }^{7}$

ille qui non desiit ridere cum hoc ipsum irati tortores omnia instrumenta crudelitatis suae experirentur. [...] plus est flamma et eculeus et lamina et vulneribus ipsis intumescentibus quod illa renovaret et altius urgueret inpressum. Inter haec tamen aliquis non gemuit. Parum est: non rogavit. Parum est: non respondit. Parum est: risit et quidem ex animo.

(Sen. epist. 78.18-19)

A ben vedere, infatti, la pena descritta è esattamente la stessa: in entrambi i casi si parla di fuoco (flamma [...] et lamina ${ }^{8}$ in Seneca; ignes candensque chalybs in Silio), quindi di allungamento degli arti, ossia del supplizio del cavalletto (eculeus in Seneca; quantum tormenta iubebant / creverunt artus in Silio) e poi ancora di ferite inferte sempre più in profondità (vulneribus ipsis intumescentibus quod illa renovaret et altius urgueret inpressum in Seneca; carnificaeve manus penitusve infusa medullis / pestis in Silio) $;{ }^{9}$ ed è lo stesso, ovviamente, anche il dettaglio della risata - è infatti questo l'elemento che, in ultima analisi, rende riconoscibile la storia anche in assenza di ogni forma di contestualizzazione.

Pur tenendo conto del fatto che questo specifico episodio si doveva essere trasformato ormai in un celebre exemplum patientiae ${ }^{10}$ - cosa che appunto permette a Seneca di parlarne in termini generici, senza fare alcun esplicito riferimento alle circostanze o ai nomi dei personaggi coinvolti -, appare comunque chiaro che la versione poetica di Silio è particolarmente vicina a quella dell'epistola 78, il che ci autorizza a postulare quantomeno una fonte comune per le due rielaborazioni, se non addirittura un consapevole recupero da parte di Silio del modello senechiano. ${ }^{11}$

6 V. in proposito Danesi Marioni (1989) e Vinchesi (2001: p. 104, n. 55).

7 Inoltre, sembrerebbe riferirsi al medesimo episodio anche un altro breve passo delle Epistole (epist. 76.20). In questo caso, però, si tratterebbe solo di un breve accenno, da cui non è possibile ricavare ulteriori informazioni sulla precisa dinamica dell'episodio.

8 La lamina è da intendersi probabilmente come una lama incandescente (cfr. Cic. Verr. 2.5.163) e corrisponde dunque pienamente al candens chalybs di Silio.

9 Cfr. Danesi Marioni (1989: pp. 248-249).

10 Cfr. Langlands (2018: pp. 219-220), la quale attribuisce un ruolo fondamentale, in questo processo, alla rielaborazione ad opera di Valerio Massimo, e mette in luce come la vicenda dello schiavo venga da quest'ultimo accostata ad altre storie esemplari, prima fra tutte il celeberrimo aneddoto di Mucio Scevola.

11 L'ipotesi è avanzata già da Danesi Marioni (1989: p. 249), la quale tuttavia fa anche notare come la più complessa argomentazione senechiana sia in realtà in parte banalizzata da Silio: per Seneca lo schiavo costituisce sì un exemplum di fortezza da imitare, ma non incarna compiutamente l'ideale del saggio stoico. 
Proprio il desiderio di emulare la vivida rappresentazione dell'epistola potrebbe infatti spiegare la particolare insistenza sul tema della tortura: con la dettagliata descrizione del supplizio, infatti, il poeta flavio sembra ricercare quelle tinte fosche già evocate dalla prosa senechiana, e a questa in genere ben più congeniali.

Inoltre, qualora si consideri la narrazione dei Punica come una rielaborazione del passo dell'epistola 78, anche la fine dell'episodio troverebbe forse una spiegazione più perspicua. Un'apparente stranezza della versione siliana è infatti costituita dal fatto che, mentre in tutte le altre fonti il focus della narrazione è sul dettaglio della risata in mezzo ai tormenti, Silio costruisce una climax di reazioni di disprezzo all'interno della quale la risata costituisce solo un gradino. Il suo personaggio, infatti, non solo supera i dolori, non solo ne ride, ma giunge addirittura a rimproverare i suoi torturatori - loro, e non lui, sfiancati dalla fatica - e alla fine chiede a gran voce di essere crocifisso come il suo padrone. E se proprio l'idea della climax sembra provenire direttamente da Seneca, il quale appunto sfruttava un analogo gioco retorico (Inter haec tamen aliquis non gemuit. Parum est: non rogavit. Parum est: non respondit. Parum est: risit et quidem ex animo), pure in Seneca la risata - in pieno accordo con le altre fonti - costituiva l'apice della climax e rimaneva dunque l'elemento simbolo della vicenda, mentre in Silio essa viene sorpassata da altre forme di disprezzo più appariscenti. Si potrebbe dunque osservare che, da bravo emulatore, qui Silio parrebbe sforzarsi in ogni modo di superare il suo modello, pur continuandone allo stesso tempo il gioco: 'ridet? Parum est!' sembrerebbe soggiungere la stessa voce fuori campo dell'epistola; e allora, se non basta, ecco che increpitat, ma di nuovo: 'Parum est!'. Ormai in tutto e per tutto trasformato in saggio stoico, ancor più senechiano del suo antecedente senechiano, lo schiavo siliano non può che dare l'estrema prova della sua patientia richiedendo a gran voce la croce del supplizio.

2. Dal supplizio e dalla stoica fermezza del servo di Tago possiamo passare ora a un'altra vicenda esemplare, ${ }^{12}$ molto simile a questa nelle sue linee essenziali, ma decisamente più nota. Questa volta si tratta della morte di Marco Atilio Regolo, avvenuta per mano dei Punici dopo che il generale romano, cui era stato concesso di tornare in patria per trattare uno scambio di prigionieri, aveva sconsigliato ai concittadini di accettare le condizioni dei nemici e aveva anzi chiesto di essere rimandato a Cartagine, mantenendo così la parola data ai perfidi Poeni. ${ }^{13}$ Eppure, anche se la vicenda è ben attestata e tramandata da numerose fonti, ${ }^{14}$ converrà osservare sin dall'inizio che ogni discorso su tale episodio

Quest'ultimo, infatti, per conseguire la virtus non può prescindere dalla ratio, elemento del tutto assente dall'aneddoto del servo. Il poeta flavio invece, dal canto suo, trasforma questo schiavo coraggioso in un vero e proprio sapiens, e dice esplicitamente che egli riesce a mantenere una mens intacta anche fra i più atroci tormenti. La ripresa del modello, insomma, sembra andare nella direzione di una semplificazione.

12 Che la vicenda di Regolo abbia valore programmaticamente esemplare, e che più nello specifico si tratti di un exemplum fidei e di un exemplum patientiae, è mostrato da von Albrecht (1964: pp. 62-68).

13 Le fonti sulla vicenda di Regolo sono numerose e non del tutto concordi l'una con l'altra: quello proposto qui è dunque da intendersi come un riassunto di servizio, basato sulla versione della vicenda offerta da Silio stesso. Per un primo orientamento sui vari filoni di tradizione si rimanda a Mix (1970) e a Gendre-Loutsch (2001).

14 Sul supplizio di Regolo v. Cantarella (2000: pp. 190-192; 317-319). Le principali fonti antiche a riguardo 
nei Punica deve necessariamente fare i conti con una difficoltà oggettiva, e cioè con il fatto che nel poema siliano la fine di Regolo è rappresentata per ben tre volte, con variazioni tutt'altro che trascurabili.

In particolare, il primo a rievocarla è il cartaginese Gestar, che, per spingere i concittadini alla guerra e per confutare la pretesa invincibilità romana, cita come esempio proprio la punizione inflitta a Regolo: uidi, cum robore pendens / Hesperiam cruce sublimis spectaret ab alta (Sil. 2.343-344). Da questo passo, dunque, sembrerebbe doversi intendere al di là di ogni ragionevole dubbio che Regolo sia stato crocifisso ${ }^{15}$ - e, peraltro, che sia stato crocifisso ancor vivo, e non post mortem, giacché la particolare drammaticità dell'immagine evocata nasce proprio dal fatto che la vittima, già innalzata sulla croce, rivolga il suo ultimo sguardo all'Italia. ${ }^{16}$ Un altro breve accenno alla morte di Regolo si trova poi poco dopo, all'interno dell'ekphrasis sullo scudo di Annibale: iuxta, triste decus, pendet sub imagine poenae / Regulus et fidei dat magna exempla Sagunto (Sil. 2.435-436). E proprio come nel passo precedente, anche qui parrebbe doversi intendere che sullo scudo fosse raffigurato un Regolo crocefisso (pendet), sebbene paradossalmente in questo caso la prospettiva appaia del tutto diversa, di matrice filo-romana: Regolo offre addirittura un exemplum fidei ai Saguntini.

La più compiuta narrazione della vicenda del console si avrà però solo nel libro sesto, ad opera del suo vecchio comes Maro, il quale riferisce a Serrano, figlio di Regolo, le gloriose ultime gesta del padre. ${ }^{17}$ Anche Maro, si noti, si qualifica come testimone oculare dei fatti (infelix vidi patriamque remissus in urbem / narrator poenae dura mercede reverti, vv. 6.529-530, in cui il vidi va inteso in senso forte, in riferimento alla punizione di Regolo a cui il vecchio aveva accennato poco prima: "io, infelice, ne fui testimone”). Eppure, in questo caso non si parla più della crocifissione di Regolo, ma di un supplizio alquanto diverso:

praefixo paribus ligno mucronibus omnes
armantur laterum crates, densusque per arcam
texitur erecti stantisque ex ordine ferri
infelix stimulus, somnisque hac fraude negatis
quocumque inflexum producto tempore torpor
inclinauit iners, fodiunt ad uiscera corpus.

(Sil. 6. 539-544)

sono: Cic. Sest. 127; Pis. 43; Phil. 11.9; fin. 2.65; 5.82-83; off. 1.39; 3.99-100; Hor. carm. 3.5, Liv. perioch. 18; Sen. dial. 1.3.9.10; 9.16.4; ben. 5.3.2; epist. 67.7; 71.17; 98.12; Val. Max. 1.1.14; 9.2. ext.1; Gell.7.4.1.

15 Anche se, come nota Fucecchi (2003: p. 278), l'espressione carceris in tenebras sembra alludere anche alla tortura del buio. E se consideriamo d'altra parte che secondo la testimonianza di Tuberone, riportata da Gellio (7.4.3), la reclusione in ambiente buio preludeva al successivo accecamento, possiamo immaginare che Gestar stia qui facendo riferimento a una pena che prevedesse dapprima la reclusione in una cella buia, quindi probabilmente l'accecamento, e da ultimo la morte in croce.

16 Per una discussione più dettagliata del problema v. Spaltenstein (1986) ad loc. Sulle modalità e sul valore della crocifissione a Roma v. Cantarella (2000: pp. 192-198); in particolare, sulla crocifissione nei Punica (sia nel passo di Regolo sia in quello del servo di Tago) v. Cotta Ramosino (1999).

17 Su questo episodio, e in particolare sulla figura di Maro, si veda Vinchesi (2006). 
Secondo il resoconto dello scudiero, quindi, Regolo sarebbe stato rinchiuso in uno strumento di tortura irto di punte acuminate che lo avrebbero poi trapassato - una versione della vicenda, dunque, abbastanza simile a quella che troviamo in Valerio Massimo:

Karthaginienses Atilium Regulum palpebris resectis machinae, in qua undique praeacuti stimuli eminebant, inclusum vigilantia pariter et continuo tractu doloris necaverunt.

(Val. Max. 9.2. ext. 1)

Mentre nel passo di Valerio è esplicitato però che è proprio tale supplizio a portare Regolo alla morte, nel caso di Silio tale informazione non viene data e il racconto di Maro è interrotto dalle lacrime di Serrano. Resta dunque aperto un problema di interpretazione tutt'altro che secondario: come conciliare questa versione con quella che si trova nel secondo libro? In effetti, l'idea che il poeta, volendo esaltare qui la patientia di Regolo di fronte ai tormenti, volontariamente non ne abbia menzionato proprio il più estremo, può sembrare strana, e la maggior parte degli interpreti ha sinora considerato i passi del libro secondo e quello del libro sesto in aperta contraddizione. ${ }^{18}$

A questo punto però, ci possiamo chiedere da che fonte provengano queste due versioni: e allora noteremo che, mentre per la versione "della botte"19 e della veglia le testimonianze sono parecchie, ${ }^{20}$ assai meno numerosi sono i testi che parlano della crocifissione. Escludendo il poema siliano, infatti, ad una crocifissione di Regolo si allude solo in Seneca e nell'Epitome di Floro.

Ora, se l'Epitome nella sua estrema sintesi ${ }^{21}$ non ci consente di capire in che modo questa variante della storia dovesse porsi rispetto alle altre, per noi di più antica attestazione, i diversi passi di Seneca risultano più interessanti. In primo luogo, possiamo infatti osservare che, delle cinque volte in cui è tirata in ballo la morte esemplare di Regolo (dial. 9.16.4; ben. 5.3.2; epist. 67.7; 71.17; 98.12), ben quattro offrono dettagli sul tipo di morte: ma anche questa volta si tratta di dettagli tra loro difficilmente compatibili.

18 Cfr. Spaltenstein (1986: ad v. 2.344) e più recentemente Fucecchi (2003: pp. 278-279), che propone anche un breve status quaestionis.

19 Per comodità, d'ora in poi con "supplizio della botte" si intenderà genericamente ogni tipo di supplizio che preveda che il condannato venga racchiuso in uno strumento ligneo irto di punte acuminate: con ciò non si vuole sostenere che lo strumento usato allo scopo sia necessariamente una botte.

20 Una narrazione piuttosto dettagliata, che raccoglie e combina tutti gli elementi tramandati dalle varie fonti, si trova in Val. Max. 9.2. ext. 1. Cicerone, nella In Pisonem (43), sostiene che la morte di Regolo sia stata causata dalla veglia prolungata, ottenuta con l'impiego di una machina, e ancora nel De Finibus (2.65; 5.82) e nel De officiis (3.100) la attribuisce alla fame e alla veglia. Gellio (7.4), per parte sua, riferisce sia la testimonianza di Tuditano, secondo cui Regolo stesso avrebbe affermato che gli era stato somministrato del veleno ad efficacia ritardata prima ancora dell'ambasceria a Roma, ma poi sarebbe stato effettivamente ucciso con la privazione del sonno, sia quella di Tuberone, secondo cui al console, tra le varie torture, sarebbero state cucite le palpebre in modo che gli occhi rimanessero aperti - e questo è un dettaglio aggiunto anche da Cicerone nella In Pisonem.

21 Alla morte di Regolo qui si accenna infatti solo molto brevemente: Nec ultimo sive carceris seu crucis supplicio deformata maiestas, 1.18.25. 
In particolare, nella versione del De tranquillitate animi - in cui Regolo è citato come esempio di morte da non compiangere - Seneca parrebbe fare riferimento al supplizio della botte: ${ }^{22}$

Neminem flebo laetum, neminem flentem: ille lacrimas meas ipse abstersit, hic suis lacrimis effecit ne ullis dignus sit. Ego Herculem fleam quod uiuus uritur, aut Regulum quod tot clauis configitur, aut Catonem quod uulnera <uulnerat> sua? Omnes isti leui temporis inpensa inuenerunt quomodo aeterni fierent, et ad inmortalitatem moriendo uenerunt.

(Sen. dial. 9.16.4)

Allo stesso modo, anche l'epistola 67 sembra perfettamente conforme alla tradizione della botte:

Tamquam opto mihi vitam honestam; vita autem honesta actionibus variis constat: in hac est $\underline{\text { Reguli }}$ arca, Catonis scissum manu sua vulnus, Rutili exilium, calix venenatus qui Socraten transtulit e carcere in caelum.

(Sen. epist. 67.7)

In questo passo Seneca, per esemplificare il caso di un desiderio implicito, cita il proposito di avere una vita virtuosa, e in seguito passa a spiegare quali possono essere le azioni che nel concreto a tale vita virtuosa conducono. A seguire inserisce dunque una serie di exempla virtutis, proponendo una breve sequenza di figure storiche insieme con l'elemento che ne ha contraddistinto la vicenda. E qui, si noti, Regolo viene accostato all'arca, termine che, tenendo conto delle varie testimonianze sulla gloriosa fine del console, non possiamo che intendere come la "botte", la cassa irta di spuntoni in cui sarebbe stato rinchiuso.

A questo punto, tuttavia, bisogna notare che all'infuori di questo passo il termine arca per lo strumento di tortura che ha provocato la morte di Regolo è usato solo - a mia conoscenza - nel tardo De viris illustribus tramandato sotto il nome di Aurelio Vittore ${ }^{23}$ e, qualora si accetti la congettura di McGushin - come fanno sia Delz, sia Fröhlich sia Vinchesi - in Silio:

densusque per arcam

texitur erecti stantisque ex ordine ferri

infelix stimulus

arcam McGushin : artem $\omega$

(Sil. 6.540-542)

Tuttavia, se consideriamo un'analoga lista di exempla nell'epistola 98, sorprendentemente, Regolo è accostato non più all'arca, ma alla croce:

22 Per quanto, secondo Stucchi (2006: p. 202), il riferimento ai chiodi in linea di principio non escluda neppure il supplizio della croce.

23 Carthaginem regressus: ubi, in arcam ligneam coniectus clavis introrsum actis, vigiliis ac dolore punitus est; 40.4. 
Dic tibi 'ex istis quae terribilia videntur nihil est invictum'. Singula vicere iam multi, ignem Mucius, crucem Regulus, venenum Socrates, exilium Rutilius, mortem ferro adactam Cato: et nos vincamus aliquid.

(Sen. epist. 98.12)

Certo, si può obiettare che crux potrebbe costituire qui un termine generico per indicare una qualche forma di tortura, ma in realtà la forza retorica di entrambe le liste nasce proprio dal fatto che i "mali" citati sono quelli che caratterizzano la particolarissima (e ben riconoscibile) vicenda di un qualche grande uomo, che vincendo un preciso, spaventosissimo tormento, lo ha reso meno temibile per la posterità. Deve essere dunque proprio il fatto di essere morto in croce, e non per qualche altra tortura, a contraddistinguere la fine di Regolo; altrimenti, nell'imprecisione, ogni valore parenetico dell'exemplum andrebbe ad attenuarsi. ${ }^{24}$ Dobbiamo immaginare dunque o che Seneca alluda a due versioni diverse della storia di Regolo - ma pure entrambe ben riconoscibili al suo lettore - oppure che faccia riferimento a due diversi momenti di una medesima storia. A ben vedere, infatti, una sorta di conciliazione delle due versioni parrebbe trovarsi nel De providentia. Qui inizialmente è descritto il supplizio della botte:

Veniamus ad Regulum: quid illi fortuna nocuit quod illum documentum fidei, documentum patientiae fecit? Figunt cutem claui et quocumque fatigatum corpus reclinauit, uulneri incumbit; in perpetuam uigiliam suspensa sunt lumina: quanto plus tormenti tanto plus erit gloriae. Vis scire quam non paeniteat hoc pretio aestimasse uirtutem? refige illum et mitte in senatum: eandem sententiam dicet.

(Sen. dial. 1.3.9-10)

Poco dopo, tuttavia, riprendendo la vicenda del console per confrontarla con quella del "delicato" Mecenate, l'autore afferma:

Mero se licet sopiat et aquarum fragoribus auocet et mille uoluptatibus mentem anxiam fallat, tam uigilabit in pluma quam ille in cruce;

(Sen. dial. 1.3.10)

A questo punto, giacché l'immediata vicinanza dei due passi ci porta a escludere la selezione da parte di Seneca di due diverse e contrastanti versioni della storia, ancora una volta la domanda che si pone è: dobbiamo credere che crux sia usato in senso generico, per indicare il dolore fisico? Oppure si tratta di due momenti diversi della medesima vicenda, l'uno successivo all'altro? Il contesto, certo, in questo caso non ci permette di escludere a priori il significato generico. Ma, pur senza voler esagerare nella pedanteria,

24 Non del tutto persuasivi mi sembrano i paralleli citati da Stucchi (2006: p. 202) per crux con il significato di "morte" o "tormento": il celeberrimo carme 85 di Catullo (Odi et amo. Quare id faciam, fortasse requiris. / Nescio, sed fieri sentio et excrucior) e un passo dell'Aulularia plautina (Quae te mala crux agitat? quid tibi mecum est commerci, senex? v. 631); in entrambi i casi, infatti, il contesto è molto più generico e non richiede quindi la stessa precisione del passo senechiano in questione - e inoltre in Plauto l'espressione mala crux è idiomatica. 
e arrivare a immaginare un Seneca cronista ed eccessivamente interessato alla veridicità storica del suo exemplum, si può forse osservare che - sia pur solo per mero artificio retorico - in realtà qui l'autore sembra sostenere che Regolo sia stato torturato con gli spuntoni non dopo, ma prima dell'ambasceria a Roma (Vis scire quam non paeniteat hoc pretio aestimasse virtutem? Refige illum et mitte in senatum: eandem sententiam dicet). Forse, allora, non è così improbabile pensare che per Seneca la vicenda di Regolo - divenuta ormai una sorta di raccolta di punizioni inusitate - potesse contenere in due momenti diversi tanto la botte quanto la croce, come già per Cicerone e per Valerio Massimo la pena della botte era andata a combinarsi con quella delle palpebre tagliate. ${ }^{25}$

Per di più, si noti, se si tiene conto del fatto che l'exemplum di Regolo parrebbe richiamato spesso per accenni, bisogna anche ammettere che Seneca doveva poter contare sul fatto che tanto l'arca quanto la crux avrebbero evocato egualmente per il suo lettore l'intera vicenda del virtuoso console. Se accettiamo tale ipotesi, dunque, possiamo considerare Seneca il più antico, anche se non certo il più coerente, testimone di una versione della storia di Regolo che lo voleva, al culmine dei suoi numerosi e svariati tormenti, morto in croce: la medesima versione cui attingeranno poi Silio (per lo meno nei due passi del secondo libro) e Floro.

Quale poi questa fonte potesse essere è pressoché impossibile da stabilire, tanto più che il lettore moderno si trova in difficoltà per la perdita della seconda decade di Livio: considerando tuttavia che in entrambi i casi la vicenda di Regolo è presentata chiaramente come exemplum virtutis, e più nello specifico come exemplum patientiae, si può forse ipotizzare che si tratti di una fonte di interesse prevalentemente morale-parenetico ${ }^{26}$ ma d'altra parte non è da escludere neppure la possibilità che si tratti di un'innovazione senechiana, ripresa poi dal poeta dei Punica.

In entrambi i casi, comunque, l'apparente incoerenza tra libro secondo e libro sesto potrebbe essere considerata come il risultato della selezione di diversi momenti della vicenda a seconda delle differenti finalità narrative. Così nel secondo libro sarebbe stato descritto unicamente il momento della morte in croce, con la potente immagine del condannato che rivolge l'ultimo sguardo alla patria perduta, mentre nel sesto libro sarebbe stato descritto soltanto il supplizio - che dobbiamo a questo punto immaginare come preliminare - della botte, in cui la patientia del comandante romano era esaltata al massimo livello: ${ }^{27}$ successivamente le lacrime di Serrano (v. 6.545) avrebbero interrotto il racconto dello scudiero prima che questi potesse arrivare a rievocare nuovamente la morte in croce.

25 Cfr. Cic. Pis. 43 e Val. Max. 9.2. ext.1.

26 È noto che fonte privilegiata per Silio, almeno per le vicende della seconda guerra punica, è Livio; se sia così anche per quelle della prima, resta per il lettore moderno un'incognita insolubile. Infatti, se si tiene conto del fatto che l'unico altro autore a citare la crux (oltre a Silio e Seneca) è Floro, un epitomatore, parrebbe doversi ipotizzare che la versione della croce risalisse in qualche modo proprio a Livio. Tuttavia, in tal caso, sarebbe ben strano che essa non abbia lasciato traccia, tra tutte le testimonianze su Regolo a noi pervenute, che in Seneca e nei Punica - e per di più, qui, solo nel secondo libro, in cui Regolo è citato solo di sfuggita, come un exemplum fidei fuori dal tempo, e non nel sesto, in cui invece alla sua fine è dedicato un lungo flashback.

27 V. Sil. 6.545-546: patientia cunctos / haec superat currus. 
3. Fino ad ora ci siamo soffermati su due celebri exempla virtutis per i quali il confronto tra il poema siliano e le opere filosofiche senechiane è stato indirizzato e facilitato proprio dall'identità della vicenda in questione. A questo punto, però, lasciando gli exempla propriamente senechiani e inoltrandoci su un terreno più lubrico possiamo porci una domanda di tipo diverso: cosa succede invece quando il nostro poeta vuole elaborare un exemplum in proprio?

Un caso di questo tipo è costituito dall'eroica morte del console Lucio Emilio Paolo durante la battaglia di Canne. Il comandante è ormai ferito quando viene raggiunto da Lentulo in fuga, il quale tenta di convincerlo a trarsi in salvo e gli offre un cavallo (Sil. 10.267-275); egli, tuttavia, lo rifiuta e dichiara di preferire la morte. L'episodio non è di invenzione siliana, e anzi mostra chiaramente di derivare dalla versione della vicenda peraltro già drammatizzata - proposta da Livio nel ventiduesimo libro (Liv. 22.49.6-9). ${ }^{28}$ Tuttavia, più di qualche tratto di originalità compare nella risposta di Paolo in Silio:

\footnotetext{
macte o uirtute paterna!

nec uero spes angustae, cum talia restent

pectora Romuleo regno. calcaribus aufer,

qua uulnus permittit, equum atque hinc ocius; urbis

claudantur portae (ruet haec ad moenia pestis)

dic, oro. rerum Fabio tradantur habenae.

nostris pugnauit monitis furor. amplius acta

quid superest uita, nisi caecae ostendere plebi

Paulum scire mori? feror an consumptus in urbem

uulneribus? quantine emptum uelit Hannibal, ut nos

uertentes terga aspiciat? nec talia Paulo

pectora, nec manes tam parua intramus imago.
}

(Sil. 10.277-288)

Se ancora l'elogio Macte o uirtute paterna! (v. 10.277) è una chiara ripresa del discorso del Paolo liviano, così come l'esortazione a mettersi in salvo quanto prima e far chiudere le porte della città (vv. 10.279-281), d'altra parte il prosieguo del discorso sembra alquanto lontano dal modello storico:

Ad ea consul: 'tu quidem, Cn. Corneli, macte uirtute esto; sed caue, frustra miserando exiguum tempus e manibus hostium euadendi absumas. abi, nuntia publice patribus urbem Romanam muniant ac priusquam uictor hostis adueniat praesidiis firment; priuatim Q. Fabio L. Aemilium praeceptorum eius memorem et uixisse [et] adhuc et mori. Me in hac strage militum meorum patere exspirare, ne aut reus iterum e consulatu sim <aut> accusator collegae exsistam ut alieno crimine innocentiam meam protegam'.

(Liv. 22.49.9-12)

28 Sul rapporto di Silio con il modello liviano - ripreso spesso molto da vicino, ma mai in modo del tutto servile -, v. Matier (1981), che si concentra in particolare sull'undicesimo libro del poema. 
Il Paolo di Livio voleva che si chiedesse pubblicamente (publice) ai senatori di fortificare la città, e inoltre che fosse riferito privatamente (privatim) a Fabio che egli si era mostrato memore dei suoi insegnamenti fino alla morte. Così, anche il Paolo di Silio consiglia di proteggere la città chiudendo le porte (claudantur portae), ma poi si discosta dal modello liviano, chiedendo direttamente che a Fabio venga affidato il comando delle operazioni militari e investendolo di fatto così (pubblicamente) come suo successore. ${ }^{29}$ Inoltre, anche le ragioni offerte per giustificare la scelta della morte appaiono, a ben vedere, alquanto diverse: nel testo dello storico il console spiegava la sua decisione argomentando che egli non voleva né essere nuovamente accusato in ragione di come aveva condotto la sua magistratura né essere costretto a farsi a sua volta accusatore del collega per difendersi, mentre il Paolo di Silio sembra assai meno interessato a proteggere Varrone e al contrario ben più preoccupato di dare un'ultima prova della propria dignità - di rendersi, insomma, con la sua morte, un exemplum virtutis per i posteri.

Le parole nostris pugnavit monitis furor, infatti, non solo costituiscono un chiaro atto d'accusa dell'operato del collega - proprio, cioè, quello che il Paolo di Livio voleva evitare -, ma pongono anche l'accento su un tema del tutto assente nello storico e che sembrerebbe avere implicazioni filosofiche: l'opposizione mens bona/furor. Paolo, in altre parole, è ben consapevole di aver messo al servizio della patria, attraverso i "moniti", la propria saggezza, che si è tuttavia dovuta scontrare col furor del collega e dei suoi seguaci. Seppure ancora in maniera implicita, dunque, il console si sta qui auto-rappresentando come sapiens, in opposizione a una non meglio determinata follia (furor) che può essere attribuita non solo a Varrone stesso, ma anche e soprattutto al popolo che lo ha sostenuto, alla caeca plebs: ${ }^{30}$ da valoroso difensore della patria pronto alla morte pur di non accusare il collega, il Paolo di Silio si sta - neanche troppo gradualmente - trasformando in una figura completamente diversa, un saggio intento a combattere, prima ancora del nemico, il furore collettivo.

$\mathrm{E}$, in effetti, l'uso del verbo ostendere è in questo senso significativo, in quanto rivela che è il console stesso a concepire come exemplum la propria fine: egli, nel rifiutare il soccorso offertogli e nell'offrirsi dunque alla morte, intende dare platealmente prova di "saper morire". A questo punto è evidente che egli non sta parlando solo da uomo politico, come in Livio, ma da sapiens.

A denunciare ormai apertamente il carattere morale dell'episodio, tuttavia, è proprio l'uso della locuzione scire mori. Questa, infatti, non può che richiamare alla memoria del lettore diversi luoghi in cui Seneca, tanto in tragedia quanto nelle opere morali, si era soffermato diffusamente su tale questione. Ad esempio, nell'Hercules Furens pressoché le stesse parole sono usate da Megara, che rivendicando la propria libertà di fronte alle minacce di Lico arriva ad affermare, in maniera mirabilmente sintetica, che solo chi non sa morire può essere soggetto a costrizione (Cogi qui potest nescit mori. Sen. HF 426). Ancora, al medesimo tema dedica una lunga riflessione il coro dell'Agamennone, per il

29 Come osserva Littlewood (2017: ad vv. 282-283) tale richiesta implica un aperto misconoscimento dell'autorità dell'altro console Varrone, e dunque risulta, di fatto, "incostituzionale".

30 Per la caratterizzazione di Varrone come demagogo si veda Ariemma (2006: in particolare pp. 223-242). 
quale proprio la capacità di ben morire rende l'uomo (o meglio, il saggio) pari a un re o persino agli dei stessi:
Solus seruitium perrumpet omne
contemptor leuium deorum,
qui uultus Acherontis atri,
qui Styga tristem non tristis uidet
audetque uitae ponere finem:
par ille regi, par superis erit.
o quam miserum est nescire mori!

(Sen. Ag. 604-610)

E soprattutto nelle opere morali però che il tema dello scire o nescire mori ricorre in modo quasi ossessivo: ad esempio, nel De brevitate vitae, la scientia vivendi ac moriendi è citata tra i beni che può garantire uno stile di vita filosofico (Sen. dial. 10.9.2); ${ }^{31}$ nel De tranquillitate animi viene invece affermato che chiunque non sappia (ben) morire è per ciò stesso destinato a vivere male (Male vivet quisquis nesciet bene mori; dial. 9.11.4). Inoltre, con parole assai simili nelle Epistole è deplorata la condizione dei più, che "non vogliono vivere, ma non sanno morire" (Plerique inter mortis metum et vitae tormenta miseri fluctuantur et vivere nolunt, mori nesciunt; epist. 4.5.5), mentre si riafferma, proprio come nell'Agamennone, che chi "sa morire" è sottratto alle ingiurie della sorte (Ego cogitem in eo qui vivit omnia posse fortunam, potius quam cogitem in eo qui scit mori nil posse fortunam? Sen. epist. 70.7.5).

Che si tratti di un tema e di un'espressione prettamente senechiana è dunque evidente, ma a tale constatazione è interessante aggiungere che proprio la medesima locuzione scire mori ricorreva anche - unica altra occorrenza nella letteratura latina a noi tramandata, all'infuori di Seneca e di Silio - in un passo di Lucano: ${ }^{32}$

\section{O felix, cui summa dies fuit obuia uicto \\ et cui quaerendos Pharium scelus obtulit enses. \\ forsitan in soceri potuisses uiuere regno. \\ scire mori sors prima uiris, sed proxima cogi.}

(Lucan. 9. 208-211)

Si tratta del discorso con cui Catone onora la fine di Pompeo, soffermandosi a considerare come, in circostanze come quelle della guerra civile, la morte costituisca la sorte migliore: e se non è possibile ottenerla con il suicidio, come sarebbe preferibile (scire mori sors prima viris), ${ }^{33}$ non è comunque da disprezzare, paradossalmente, l'esservi costretti.

31 L'espressione alta rerum quies è peraltro molto vicina a quella usata poco prima nel coro dell'Agamennone (pax alta, v. 195): e in effetti i due passi, particolarmente affini, sembrano esprimere il medesimo concetto.

32 L'eco lucanea è notata dalla Littlewood (2017: ad vv. 10.283-285).

33 Incidentalmente si può osservare che tale massima lucanea sembra modellata su un passo senechiano: solus te iam praestare potest / furor insontem: proxima puris / sors est manibus nescire nefas (HF 1097-1099). 
Ora, tale riflessione assume chiaramente un valore del tutto particolare per il fatto che ad esprimerla è Catone, cioè la figura che tanto nel poema lucaneo quanto nella riflessione filosofica senechiana incarna nella maniera più compiuta l'ideale del saggio stoico. ${ }^{34}$ Proprio per questo, è interessante notare come nei Punica l'espressione usata dal Catone di Lucano sia ripresa in un episodio che si pone come idealmente intermedio tra quello di Pompeo e quello di Catone: la fine di Paolo, morto sul campo di battaglia, infatti sarebbe da assimilare alla morte in Egitto del primo, piuttosto che all'eroico suicidio del secondo. Eppure, l'orgogliosa rivendicazione di "saper morire", che implica un'adesione piena e volontaria a un destino già determinato, in linea con la concezione stoica, ha l'effetto di trasformare un atto di violenza e un'imposizione (Paolo è in realtà coactus) in una scelta - e una scelta che normalmente deriva da una precisa formazione filosofica, vale a dire quella stoica. Ecco, dunque, che il Paolo di Silio, attraverso la consapevole decisione di rifiutare il soccorso di Lentulo, può attingere a quella stessa sors prima che Lucano riservava al suo Catone, al di là delle circostanze contingenti che ne causano la morte, in base alle quali egli dovrebbe essere, semmai, un Pompeo. ${ }^{35}$

Inoltre, molto significativa in tal senso risulta anche la rivendicazione nec manis tam parva intramus imago. Se infatti a un primo livello si può senz'altro tradurre, con la Vinchesi, «non è un'ombra tanto meschina quella che sta per entrare nella dimora dei Mani», e cogliere in tale affermazione, insieme con Spaltenstein, un'eco delle estreme parole della Didone virgiliana (magna mei sub terra ibit imago; Verg. Aen. 4.654), d'altra parte è pur vero che imago è termine almeno in parte ambiguo. Imagines sono infatti le effigi di cera degli antenati che venivano conservate dalle famiglie illustri, e in poesia imagines sono dette le ombre dei morti, ${ }^{36}$ ma imago può indicare anche l'immagine intesa come figura e allegoria, e in particolare virtutium viva imago è definito da Seneca proprio Catone nel momento in cui se ne rievoca la morte esemplare (Sen. dial. 9.16.1).

Dunque, quando il poeta flavio fa affermare al suo Paolo di voler mostrare alla folla che egli "sa morire", e per di più gli fa esplicitamente dichiarare l'intenzione di scendere nell'oltretomba come non parva imago, in tali parole si potrà forse leggere, oltre all'evidente ripresa del modello mitico di Didone - certo esempio anch'essa di morte onorevole, ma in questo caso del tutto fuori contesto - l'intenzione di trasformare anche Paolo in una virtutium viva imago paragonabile a Catone, l'esempio paradigmatico offerto più e più volte da Seneca della capacità di ben morire.

34 Non è del resto un caso che a Catone, vero sapiens, toccherà proprio quella sors prima, mentre a Pompeo, cittadino utile ma comunque non del tutto esente da mende e pertanto non comparabile agli antichi, la sors proxima di una morte inflitta dal nemico (cfr. Lucan. 9.190-191).

35 Su un piano letterario, quindi, Paolo nei Punica recepirebbe il modello di Catone, pur essendone in realtà un predecessore sul piano storico, e insieme con lui andrebbe a inserirsi nella serie di coloro la cui morte è diventata per i posteri exemplum virtutis. 


\section{Conclusioni}

Nel complesso, dunque, parrebbe di poter affermare che Silio guardasse con notevole interesse a Seneca, e in particolare agli exempla virtutis di cui l'opera morale di Seneca è costellata. Non solo: proprio l'impiego di lessico e motivi spiccatamente senechiani sembra marcare quei luoghi del poema siliano in cui la matrice filosofica stoica vuole essere esplicitamente esibita, discussa e problematizzata. È così nel sesto libro, in cui l'imperturbabile Regolo, eroe della generazione precedente, viene evocato come guida morale per il figlio Serrano, ma anche nel meno noto episodio del primo libro in cui la fermezza del servo di Tago serve da contrappunto per mettere in luce la furiosa crudeltà dei Cartaginesi.

Il modello senechiano, tuttavia, può anche essere sfruttato come una sorta di repertorio di temi e motivi attraverso cui un episodio storico come la morte di Paolo a Canne, che con la filosofia aveva originariamente ben poco a che fare, viene trasformato dal poeta dei Punica in un nuovo exemplum virtutis tutto romano, fuso con la materia stessa del poema. E per di più in questo caso siamo di fronte a un personaggio che non si limita a morire eroicamente, ma vuole consapevolmente "mostrare" (ostendere) di "saper morire", attribuendo dunque programmaticamente alla propria fine un valore esemplare.

Non sarà dunque casuale la ripresa esplicita né del motivo senechiano del saper/non saper morire, né della tessera lucanea (scire mori) con la quale tale motivo era già stato da qualche decennio trasposto nell'epica storica. Il Paolo di Silio, insomma, vuole sì insegnare ai suoi concittadini a "ben morire", ma prima - si direbbe - lo ha imparato lui stesso, andando a scuola (intertestualmente) dal maestro migliore, cioè da quel Catone Uticense di cui Seneca e Lucano avevano fatto forse la più compiuta incarnazione della figura del sapiens nella storia romana.

\section{Bibliografia}

Ariemma, E. M. (2006). Tentazioni demagogiche nei Punica di Silio Italico. Aevum Antiquum, 6, 217-242.

Bassett, E. L. (1966). Hercules and the Hero of the Punica. In L. Wallach (Ed.), The Classical Tradition: Literary and Historical Studies in Honor of Harry Caplan (pp. 258-273). Ithaka: Cornell University Press.

Billerbeck, M. (1986a). Aspects of Stoicism in Flavian Epic. In F. Cairns (Ed.), Papers of the Liverpool Latin Seminar (Vol. 5; pp. 341-356). Liverpool: Francis Cairns (Publications).

Billerbeck, M. (1986b). Stoizismus in der römischen Epik neronischer und flavischer Zeit. In W. Haase (Ed.), Aufstieg und Niedergang der Römischen Welt. Sprache und Literatur (Literatur der julischclaudischen und der flavischen Zeit [Forts.]) (Vol. II. 32.5; pp. 3116-3151). Berlin - New York: Walter de Gruyter.

Cantarella, E. $\left(2000^{3}\right)$. I supplizi capitali in Grecia e a Roma. Origine e funzioni delle pene di morte nell'antichità classica. Milano: Rizzoli.

Cotta Ramosino, L. (1999). Il supplizio della croce in Silio Italico (Pun. I 169-181; VI 539-544). Aevum, 73, 93-106. 
Danesi Marioni, G. (1989). Un martirio stoico: Silio Italico, Pun. 1.169 ss. Prometheus, 15, 245-253.

Delz, J. (Ed.). (1987). Sili Italici Punica (Bibliotheca Scriptorum Graecorum et Romanorum Teubneriana). Stutgardiae: Walter de Gruyter.

Frölich, U. (2000). Regulus, Archetyp römischer Fides. Das sechste Buch als Schlüssel zu den Punica des Silius Italicus. Interpretation, Kommentar und Übersetzung. Tübingen: Stauffenburg Verlag.

Fucecchi, M. (2003). I Punica e altre storie di Roma nell'Epos di Silio Italico. In A. Casanova, \& P. Desideri (Eds.), Evento, racconto, scrittura nell'antichità classica. Atti del Convegno internazionale di studi (Firenze, 25-26 novembre 2002) (pp. 269-292). Firenze: Università degli Studi di Firenze, Studi e Testi.

Gazich, R. (2003). Retorica dell'esemplarità nelle Lettere di Plinio. In L. Castagna, \& E. Lefèvre (Eds.), Plinius der Jüngere und seine Zeit (pp. 123-141). München - Leipzig: K. G. Saur.

Gendre, M., \& Loutsch, C. (2001). C. Duilius et M. Atilius Regulus. In M. Coudry, \& T. Späth (Eds.), L'invention des grands hommes de la Rome antique. Actes du colloque du Collegium Beatus Rhenanus, Augst, 16-18 septembre 1999 (pp. 131-172). Paris: de Boccard.

Hölkeskamp, H.-J. (1996). Exempla und mos maiorum. Überlegungen zum kollektiven Gedächtnis der Nobilität. In H.J. Gehrke, \& A. Möller (Eds.), Vergangenheit und Lebenswelt. Soziale Kommunikation, Traditionsbildung und historisches Bewußtsein (pp. 301-338). Tübingen: Gunter Narr Verlag.

Langlands, R. (2018). Exemplary Ethics in Ancient Rome. Cambridge: Cambridge University Press.

Littlewood, J. (2017). A Commentary on Silius Italicus' Punica 10. Oxford: Oxford University Press.

Matier, K. O. (1981). Prejudice and the Punica: Silius Italicus - A Reassessment. Acta Classica, 24, $141-151$.

Matier, K. O. (1990). Stoic Philosophy in Silius Italicus. Akroterion, 35, 68-72.

Mix, E. R. (1970). Marcus Atilius Regulus: Exemplum Historicum. The Hague: Mouton.

Reynolds, L. D. (Ed.). (1965). L. Annaei Senecae Ad Lucilium Epistulae Morales, tomus I (libri I-XIII), II (libri XIV-XX). Oxford: Oxford University Press.

Reynolds, L. D. (Ed.). (1977). L. Annaei Senecae Dialogorum Libri Duodecim. Oxford: Oxford University Press.

Spaltenstein, F. (1986). Commentaire des Punica de Silius Italicus, livres 1 à 8. Genève: Librairie Droz S. A.

Spaltenstein, F. (1990). Commentaire des Punica de Silius Italicus, livres 9 à 17. Genève: Librairie Droz S. A.

Stemmler, M. (2000). Auctoritas exempli. Zur Wechselwirkung von kanonisierten Vergangenheitsbildern und gesellschaftlicher Gegenwart in der spätrepublikanischen Rhetorik. In B. Linke, \& M. Stemmler (Eds.), Mos maiorum: Untersuchungen zu den Formen der Identitätsstiftung und Stabilisierung in der römischen Republik (pp. 141-205). Stuttgart: Franz Steiner Verlag.

Stucchi, S. (2006). Estetica dell'agonia: la rappresentazione di dolore e tormento in Silio e il caso di Regolo. Aevum Antiquum, 6, 197-213.

Tipping, B. (2010). Exemplary Epic. Silius Italicus' Punica. Oxford: Oxford University Press.

Vinchesi, M. A. (Transl.). (2001). Silio Italico, Le guerre puniche. Milano: Rizzoli.

Vinchesi, M. A. (2006). Maro e l'incontro con il figlio di Regolo: la tipologia di un personaggio minore nel VI libro dei Punica. Aevum Antiquum, 6, 257-272. 
von Albrecht, M. (1964). Silius Italicus: Freiheit und Gebundenheit römischer Epik. Amsterdam: Verlag P. Schippers.

von Albrecht, M. (1999). Roman Epic. An Interpretative Introduction. Leiden - Boston - Köln: Brill. Zwierlein, O. (Ed.). (1986). L. Annaei Senecae Tragoediae. Incertorum auctorum Hercules [Oetaeus], Octavia. Oxford: Oxford University Press.

Diletta Vignola / diletta.vignola@sns.it

Department of Italian Studies, Romance Studies, Classics, Arts and Drama Studies

University of Genoa, School of Humanities

DIRAAS, via Balbi 2, 16124 Genova, Italy 
